\section{Perfil sócio-demográfico e epidemiológico dos trabalhadores da atenção básica à saúde nas regiões Sul e Nordeste do Brasil}

\author{
Epidemiological and socio-demographic profile \\ of primary care workers in the South \\ and Northeast of Brazil
}

\author{
1 Universidade Católica de \\ Pelotas, Pelotas, Brasil. \\ 2 Secretaria Municipal de \\ Saúde e Bem Estar, Pelotas, \\ Brasil. \\ 3 Programa de Pós-graduação \\ em Epidemiologia, \\ Universidade Federal de \\ Pelotas, Pelotas, Brasil. \\ 4 Faculdade de Enfermagem \\ e Obstetrícia, Universidade \\ Federal de Pelotas, Pelotas, \\ Brasil. \\ Correspondência \\ E. Tomasi \\ Universidade Católica \\ de Pelotas. \\ Rua Marechal Deodoro 1078, \\ apto. 304, Pelotas, RS \\ 96020-220, Brasil. \\ tomasiet@uol.com.br
}

\begin{abstract}
In order to describe the profile of primary health care teams in 41 municipalities with more than 100 thousand inhabitants each, a total of 4,749 health workers in two States from the South $(1,730)$ and five from the Northeast $(3,019)$ of Brazil were included from a sample of traditional primary care units and the Family Health Program (FHP). After providing informed consent, the health workers answered a self-applied questionnaire with demographic, work-related, and their own health-related data. The principal differences between the two models involved the structuring of the teams, with the FHP including more community health agents, more women, more young workers, fewer hired on the basis of formal admissions exams, more with a single job, more precarious employment arrangements, less employment satisfaction, less time on the job, larger workloads, greater specialization in the area, and better pay. The FHP also showed worse self-perceived health and more medical appointments. Management efforts are needed to support these workers, who form the basis of the health system and are key protagonists in the development and consolidation of primary care.
\end{abstract}

Primary Health Care; Family Health; Occupational Health; Working Conditions
Elaine Tomasi 1,2

Luiz Augusto Facchini 3

Roberto Xavier Piccini 3

Elaine Thumé 4

Denise Silva da Silveira 2,3

Fernando Vinholes Siqueira 2,3

Maria Aparecida Rodrigues 2,3

Vera Vieira Paniz 3

Vanessa Andina Teixeira 3

\section{Introdução}

O impacto do trabalho sobre a saúde tem sido investigado com regularidade em diversas categorias profissionais $1,2,3$. Com o crescimento mundial do setor de serviços, os trabalhadores de saúde tornaram-se um dos maiores contingentes da força de trabalho. Essencial para a vida humana, o trabalho em saúde insere-se na esfera da produção imaterial, que se completa no ato de sua realização. Ele é coletivo, contando com a participação de diversos trabalhadores e envolve características tanto em sua forma assalariada e fragmentada, quanto artesanal 4.

Estudos sobre o trabalho em saúde devem considerar a complexidade das demandas em saúde, as relações entre as equipes e a articulação dos diferentes trabalhos em uma equipe de saúde, determinando possibilidades e limites de respostas às necessidades de saúde das pessoas 5,6. O desgaste profissional, a satisfação no trabalho e a morbidade psíquica entre médicos de atenção primária vêm sendo estudados por diversos autores $7,8,9$, tendo sido detectadas elevadas prevalências de síndrome de burnout (de 25\% a 70\%).

No âmbito do Programa Saúde da Família (PSF) 10 sabe-se que atividades concernentes à gerência das unidades básicas de saúde e (UBS) representam acúmulo de trabalho. Vínculos de trabalho precários geram insegurança, dificuldade no gozo dos direitos trabalhistas e reivindicações quanto ao reconhecimento profissional. 
Embora os trabalhadores da atenção básica constituam um enorme contingente de força de trabalho, no contexto do SUS e do país, pouco se sabe sobre suas condições de trabalho e saúde. Acredita-se que esses profissionais são a base do sistema de saúde e, portanto, protagonistas do desenvolvimento e melhoria deste sistema 11 . Seu processo de trabalho é bastante peculiar, no qual interagem habilidades técnicas e relações interpessoais, além do compromisso implícito e para muitos desconhecido, com o entender coletivo do processo saúde-doença, recaindo sobre eles grandes e crescentes responsabilidades.

O Projeto de Expansão e Consolidação do Saúde da Família (PROESF) é uma iniciativa do Ministério da Saúde, dirigida ao fortalecimento da Atenção Básica à Saúde, particularmente em municípios com mais de 100 mil habitantes 12 .

A avaliação dos perfis profissionais, das condições de trabalho e desgaste ocupacional pode subsidiar programas de readequação de processos de trabalho, com o objetivo de melhorar o atendimento da população.

O objetivo deste estudo é avaliar o perfil das equipes de saúde da rede básica em 41 municípios com mais de 100 mil habitantes de sete estados das Regiões Sul e Nordeste, de acordo com características demográficas, da formação profissional, do trabalho e da situação de saúde física e psíquica.

\section{Métodos}

O universo do estudo foi constituído por $41 \mathrm{mu}$ nicípios com mais de 100 mil habitantes, assim distribuídos: 17 do Rio Grande do Sul e quatro de Santa Catarina (Lote 2 Sul); dois em Alagoas, três na Paraíba, dez em Pernambuco, dois no Piauí e três no Rio Grande do Norte (Lote 2 Nordeste) 12. Os trabalhadores foram incluídos a partir da amostra das UBS segundo o modelo de atenção (PSF ou tradicional) e proporcionalmente ao total de UBS nos municípios 13,14,15. Por meio dos Projetos de Adesão ao PROESF, foram identificadas 855 UBS no Nordeste e 655 UBS no Sul. Em cada lote foram sorteadas aleatoriamente 120 UBS. No Sul, a amostra foi composta por 69 UBS do PSF e 51 tradicionais, enquanto no Nordeste 79 eram do PSF e 41 tradicionais. Todos os trabalhadores das 240 UBS foram convidados a participar do estudo, incluindo médicos, enfermeiros, nutricionistas, odontólogos, psicólogos, técnicos e auxiliares de enfermagem, recepcionistas e agentes comunitários de saúde.

A coleta de dados ocorreu entre os meses de março e agosto de 2005, com uma equipe de 15 auxiliares de pesquisa (entrevistadores) vincula- dos ao Estudo de Linha de Base de Avaliação do PROESF 16. Após consentimento, os trabalhadores responderam a um questionário auto-aplicado constituído de informações demográficas, sócio-econômicas, características do trabalho e de situação de saúde. A avaliação de condições estressoras no ambiente de trabalho 17 incluiu aspectos do ambiente físico, fatores próprios das tarefas, aspectos institucionais e pessoais. A aferição dos transtornos mentais comuns foi realizada usando-se o Self-Report Questionnaire-20 (SRQ-20) 18. Digitados no programa Epi Info 6.04b (Centers for Disease Control and Prevention, Atlanta, Estados Unidos), os bancos de dados foram exportados através do aplicativo Stat Transfer 5.0 (Circle Systems, Seattle, Estados Unidos; http://www.stattransfer.com/index. html) para o pacote estatístico SPSS 10.0 (SPSS Inc., Chicago, Estados Unidos). Inicialmente, procedeu-se às análises descritivas, verificando a distribuição dos casos em cada variável. A análise bivariada examinou o comportamento das variáveis segundo o modelo de atenção (PSF e tradicional) e a região (Sul e Nordeste).

Para as variáveis dependentes qualitativas as associações foram testadas por meio da comparação entre proporções, utilizando-se o teste do qui-quadrado. No caso de variáveis dependentes do tipo quantitativas, as associações foram testadas usando-se a comparação entre médias, utilizando-se o teste F (ANOVA). Para ambos, foram consideradas significativas as diferenças com valor de p inferior a 0,05 .

O estudo foi apreciado pelo Comitê de Ética da Universidade Federal de Pelotas, tendo obedecido todos os requisitos para sua aprovação.

\section{Resultados}

Foram estudados 4.749 trabalhadores de saúde, 1.730 no Sul e 3.019 no Nordeste. A Figura 1 apresenta a distribuição da amostra de acordo com a função desempenhada no serviço de saúde, a região e o modelo de atenção. Chama atenção a maior proporção de médicos em UBS tradicionais no Sul e a expressiva presença de agentes comunitários de saúde no Nordeste, tanto no PSF quanto na atenção tradicional.

A renda bruta mensal referida pelos trabalhadores, de acordo com a sua formação ou nível de escolaridade, foi relacionada com o emprego na UBS. Comparando-se os dois lotes, as médias salariais para os médicos ( $\mathrm{p}<0,001)$, os demais profissionais de níveis superior ( $\mathrm{p}<0,001)$ e médio ( $\mathrm{p}=0,03)$ e os auxiliares de enfermagem ( $\mathrm{p}<$ 0,001 ) foram significativamente maiores no Sul do que no Nordeste, não diferindo para os en- 

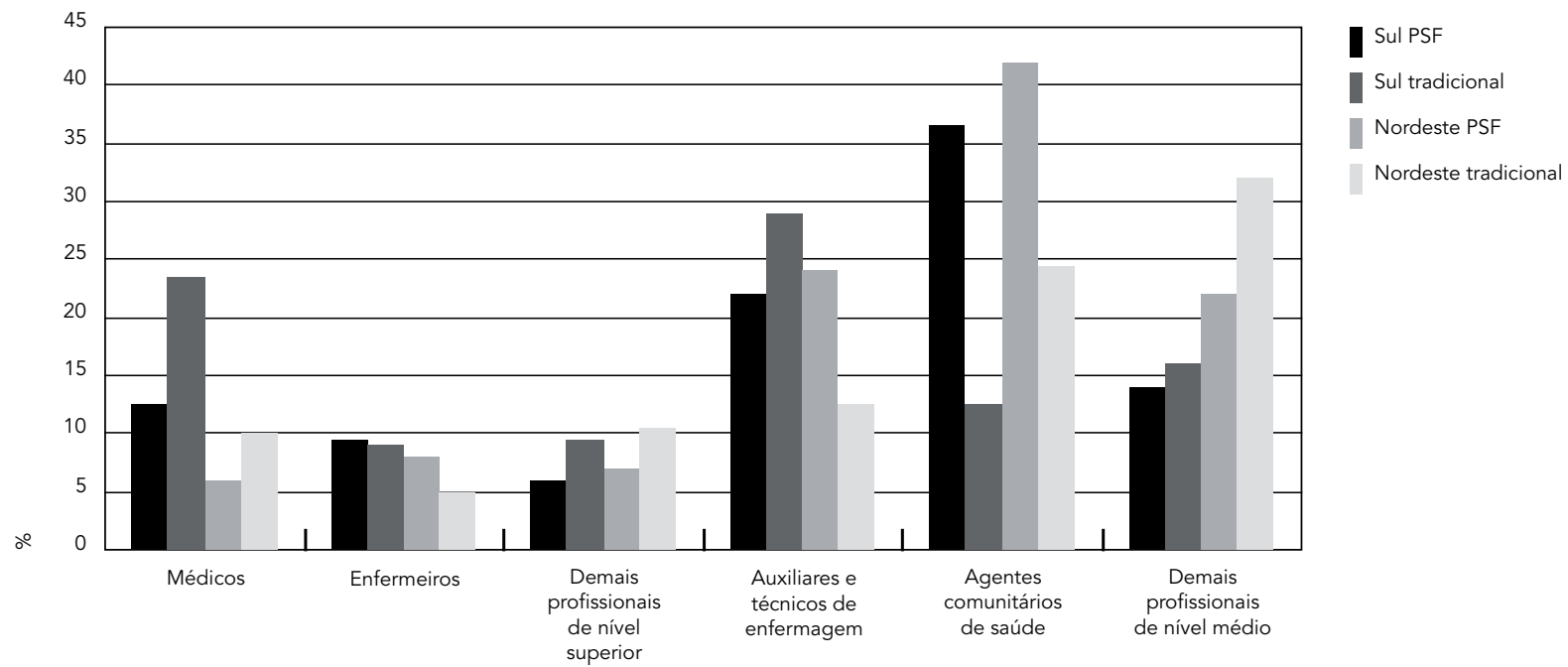

PSF: Programa Saúde da Família.

fermeiros $(p=0,24)$ e agentes comunitários de saúde $(\mathrm{p}=0,90)$. Observou-se ainda que a média salarial foi superior nas UBS do PSF, exceto para os demais trabalhadores de nível médio do Sul $(\mathrm{p}=0,28)$ e do Nordeste $(\mathrm{p}=0,30)$ e os agentes comunitários de saúde do Nordeste $(\mathrm{p}=0,45)$ (Tabela 1).

A amostra foi formada por 3.790 (81\%) trabalhadores do sexo feminino e 885 (19\%) do masculino. A média de idade dos trabalhadores foi de 37 anos (DP = 10,4) no Sul e de $38(\mathrm{DP}=10,3)$ no Nordeste. A maior proporção de trabalhadores se concentrou no grupo etário de 31 a 45 anos de idade para os dois modelos de atenção. A proporção de trabalhadores concentrados na categoria de idade até trinta anos foi maior para o PSF, enquanto na categoria de 46 anos e mais a situação se inverteu, ou seja, os trabalhadores do PSF são significativamente mais jovens $(\mathrm{p}<0,001)$ do que os vinculados ao modelo tradicional, em ambos os lotes (Tabela 2).

Pouco menos de $20 \%$ dos profissionais entrevistados no Sul e cerca $30 \%$ no Nordeste fizeram referência ao trabalho atual como primeiro emprego, sendo significativa esta diferença entre os lotes $(p<0,001)$. A proporção média de trabalhadores com único emprego foi semelhante entre os lotes, sendo significativamente maior entre aqueles vinculados ao PSF em ambos os lotes (Tabela 2).
O ingresso por concurso público alcançou pouco mais de $40 \%$ dos trabalhadores da rede básica, sendo maior no modelo de atenção tradicional do que no PSF tanto no Sul quanto no Nordeste (Tabela 2).

$\mathrm{O}$ vínculo de trabalho tipicamente precário (sem garantias trabalhistas) alcançou aproximadamente $38 \%$ dos trabalhadores da atenção básica e foi significativamente maior entre os entrevistados no Nordeste (42\%) em relação aos estudados no Sul (30\%). A precarização do trabalho no Lote Sul foi independente do modelo de atenção, enquanto no Nordeste foi superior para os profissionais das UBS do PSF (48\%) em relação ao modelo tradicional (33\%) (Tabela 2). Aproximadamente dois terços dos profissionais estavam satisfeitos com o vínculo de trabalho, significativamente $(\mathrm{p}<0,001)$ mais no Sul $(69 \%)$ do que no Nordeste (58\%). Em ambas as regiões, os profissionais das UBS tradicionais estavam mais satisfeitos com o vínculo de trabalho do que seus colegas do PSF.

A maioria dos trabalhadores $(48 \%)$ estava vinculada à UBS há mais de dois anos, e esta proporção foi superior no Nordeste (55\%) quando comparada com o Sul (37\%). Independente do lote, o tempo de trabalho na UBS foi em média maior nas UBS tradicionais do que nas do PSF. 
Renda média mensal $(\mathrm{R} \$$ ) dos trabalhadores em saúde, por categoria profissional, modelo de atenção e lote. Estudo de Linha de Base, Projeto de Expansão e Consolidação do Saúde da Família, Universidade Federal de Pelotas, 2005.

\begin{tabular}{|c|c|c|c|c|c|c|}
\hline \multirow[t]{3}{*}{ Categoria profissional } & \multicolumn{3}{|c|}{ Lote 2 Sul (R\$) } & \multicolumn{3}{|c|}{ Lote 2 Nordeste (R\$) } \\
\hline & PSF & Tradicional & Total & PSF & Tradicional & Total \\
\hline & Média (DP) & Média (DP) & Média (DP) & Média (DP) & Média (DP) & Média (DP) \\
\hline Médicos & $3.713(1.509)$ & $1.898(1.005)$ & $2.750(1.556)$ & $3.084(1.182)$ & $1.097(755)$ & $2.128(1.409)$ \\
\hline Enfermeiros & $2.211(788)$ & $1.644(922)$ & $2.050(864)$ & 2.399 (789) & $1.504(804)$ & $2.173(881)$ \\
\hline Demais profissionais de nível superior & $2.608(1.260)$ & $1.994(1.264)$ & $2.326(1.293)$ & $2.163(903)$ & $920(365)$ & $1.529(924)$ \\
\hline Auxiliares e técnicos de enfermagem & $931(598)$ & $748(394)$ & $859(534)$ & $646(338)$ & $436(193)$ & $554(302)$ \\
\hline Demais profissionais de nível médio & 512 (399) & $574(396)$ & $534(398)$ & $486(375)$ & $458(342)$ & $473(361)$ \\
\hline Agentes comunitários de saúde & $387(88)$ & $359(60)$ & $382(85)$ & $388(285)$ & 372 (329) & $384(297)$ \\
\hline
\end{tabular}

PSF: Programa Saúde da Família.

O contrato de trabalho de quarenta horas semanais incluiu mais de dois terços dos trabalhadores do PSF, enquanto no modelo tradicional esta proporção alcançou menos da metade dos trabalhadores, nas duas regiões.

Entre os profissionais de nível superior, somente $37 \%$ tinham especialização, sem diferença significativa entre os lotes ( $\mathrm{Sul}=35 \%$ e Nordeste $=39 \%$ ). Entretanto, em ambos os lotes os profissionais do PSF informaram possuir especialização na área em proporções que alcançaram o dobro daquela informada por profissionais do modelo tradicional.

Menos de $70 \%$ dos trabalhadores referiram receber supervisão no desempenho de suas atividades, a ocorrência de supervisão semanal foi informada por um terço destes, com diferença significativa entre os lotes, sendo de $35 \%$ no Sul e de $27 \%$ no Nordeste $(\mathrm{p}<0,001)$. É interessante a constatação de que a proporção de supervisão semanal foi maior nas UBS do PSF no Sul e nas UBS tradicionais do Nordeste (Tabela 2). Independente da região e do modelo, o conteúdo de supervisão mais citado foi o monitoramento da produtividade (33\%) e das rotinas diárias $(13 \%$ no Sul e $20 \%$ no Nordeste), seguido de orientações gerais ( $10 \%$ no Sul e $13 \%$ no Nordeste).

Quase metade dos trabalhadores considerou o ambiente físico das UBS inadequado (46\%), sendo $43 \%$ no Sul e $48 \%$ no Nordeste $(p=0,001$ ). Os trabalhadores mais insatisfeitos com o ambiente físico foram aqueles das UBS tradicionais do Nordeste. A avaliação de inadequação das tarefas foi registrada para $43 \%$ da amostra, sendo significativamente diferente entre os lotes: $36 \%$ no Sul e $47 \%$ no Nordeste ( $p<0,001)$. Em ambas as regiões a insatisfação em relação à adequação de tarefas foi maior entre trabalhadores vinculados ao PSF. Mais de 30\% dos entrevistados refe- riram inadequação das relações institucionais, marcadamente $(\mathrm{p}<0,001)$ menos no Nordeste (17\%) do que no Sul (56\%), embora nos dois lotes esta proporção tenha sido significativamente superior entre os trabalhadores do PSF. O último item da avaliação das condições de trabalho, que considera as relações pessoais, foi julgado inadequado por mais de um terço da amostra no Lote Sul, sem diferença entre modelos de atenção. No Lote Sul, mais de $90 \%$ da amostra acusaram inadequação das relações pessoais, sendo ainda significativamente maior esta proporção entre os trabalhadores do PSF do Nordeste (95\%).

Um terço dos trabalhadores declarou-se um pouco ou muito insatisfeito com sua saúde, significativamente mais no Sul (37\%) do que no Nordeste (31\%). O Sul também apresentou diferenças importantes de acordo com o modelo de atenção: $39 \%$ entre profissionais do PSF e $32 \%$ do modelo tradicional.

Problemas de saúde foram referidos por $42 \%$ dos trabalhadores, sendo menor a prevalência ( $\mathrm{p}<0,001)$ no Sul $(38 \%)$ do que no Nordeste (45\%). De acordo com os capítulos da Classificação Internacional de Doenças 10a Revisão (CID-10), os principais problemas entre os trabalhadores foram as doenças do aparelho circulatório ( $25 \%$ no Sul e $26 \%$ no Nordeste), as do sistema osteomuscular e do tecido conjuntivo (13\% no Sul e Nordeste) e as doenças do aparelho digestivo ( $11 \%$ no Sul e $15 \%$ no Nordeste).

O uso regular de medicamentos foi informado por um quarto $(26 \%)$ da amostra de trabalhadores, que representa $67 \%$ dos profissionais que referiram algum problema de saúde, e esta informação não revelou diferenças significativas entre os lotes.

A automedicação foi referida por $20 \%$ de todos os trabalhadores, um pouco mais no Sul 
Características demográficas, do trabalho e da saúde dos profissionais por modelo de atenção e Lote. Estudo de Linha de Base, Projeto de Expansão e Consolidação do Saúde da Família, Universidade Federal de Pelotas, 2005.

\begin{tabular}{|c|c|c|c|c|c|c|}
\hline \multirow[t]{3}{*}{ Variável } & \multicolumn{3}{|c|}{ Lote Sul } & \multicolumn{3}{|c|}{ Lote Nordeste } \\
\hline & PSF & Tradicional & $\mathrm{p}$ & PSF & Tradicional & $\mathrm{p}$ \\
\hline & $\%(n)$ & $\%(n)$ & & $\%(n)$ & $\%(n)$ & \\
\hline \multicolumn{7}{|l|}{ Características demográficas } \\
\hline Sexo feminino & $86(936)$ & $76(473)$ & $<0,001$ & $81(.1480)$ & $80(901)$ & 0,4 \\
\hline Idade (anos) & & & $<0,001$ & & & $<0,001$ \\
\hline$\leq 30$ & $36(396)$ & $22(137)$ & & $30(541)$ & $21(236)$ & \\
\hline $31-45$ & $45(495)$ & $49(306)$ & & $50(915)$ & $47(536)$ & \\
\hline $46 e+$ & $19(202)$ & $29(178)$ & & $20(363)$ & $32(365)$ & \\
\hline \multicolumn{7}{|l|}{ Características do trabalho } \\
\hline Ingresso por concurso & $34(363)$ & $59(358)$ & $<0,001$ & $36(653)$ & $44(493)$ & $<0,001$ \\
\hline Primeiro emprego & $18(192)$ & $18(111)$ & 0,87 & $31(569)$ & $34(383)$ & 0,2 \\
\hline Único emprego & 85 (931) & $71(432)$ & $<0,001$ & $83(1.523)$ & $73(832)$ & $<0,001$ \\
\hline Vínculo precário de trabalho & $30(325)$ & $28(168)$ & 0,45 & 48 (854) & $33(371)$ & $<0,001$ \\
\hline Satisfação com vínculo de trabalho & $65(699)$ & $78(464)$ & $<0,001$ & $50(915)$ & $71(806)$ & $<0,001$ \\
\hline Tempo de trabalho na UBS (meses) & & & $<0,001$ & & & $<0,001$ \\
\hline$\leq 6$ & $23(249)$ & $22(130)$ & & $10(174)$ & $11(125)$ & \\
\hline $7-12$ & $27(296)$ & $14(83)$ & & $20(355)$ & $13(144)$ & \\
\hline $13-24$ & $20(212)$ & $13(79)$ & & $20(360)$ & $14(159)$ & \\
\hline 25 ou + & $30(327)$ & $51(301)$ & & $50(915)$ & $62(679)$ & \\
\hline Contrato de 40 horas semanais & $84(913)$ & $49(288)$ & $<0,001$ & $88(1.577)$ & $47(518)$ & $<0,001$ \\
\hline Especialização na área * & $46(100)$ & $22(38)$ & $<0,01$ & $45(123)$ & $28(40)$ & 0,001 \\
\hline Supervisão semanal do trabalho & $38(280)$ & $28(63)$ & 0,008 & $19(237)$ & $44(240)$ & $<0,001$ \\
\hline \multicolumn{7}{|l|}{ Condições de trabalho inadequadas } \\
\hline Ambiente físico & $42(461)$ & $44(277)$ & 0,36 & $44(823)$ & $53(622)$ & $<0,001$ \\
\hline Tarefas & $41(456)$ & $25(159)$ & $<0,001$ & $54(1.007)$ & $35(405)$ & $<0,001$ \\
\hline Relações institucionais & $60(656)$ & $50(314)$ & $<0,001$ & $17(308)$ & $19(215)$ & 0,21 \\
\hline Relações pessoais & $95(1.049)$ & $92(578)$ & 0,011 & $9(172)$ & $16(180)$ & $<0,001$ \\
\hline \multicolumn{7}{|l|}{ Situação de saúde } \\
\hline Insatisfação com a saúde & $39(426)$ & $32(196)$ & 0,006 & $30(558)$ & $31(359)$ & 0,63 \\
\hline Algum problema de saúde & $39(426)$ & $34(209)$ & 0,05 & $45(759)$ & $45(491)$ & 0,8 \\
\hline Uso regular de medicamentos ** & $69(293)$ & $71(145)$ & 0,65 & $63(474)$ & $69(330)$ & 0,04 \\
\hline Automedicação & $18(185)$ & $28(156)$ & $<0,001$ & $16(283)$ & $23(237)$ & $<0,001$ \\
\hline Transtornos mentais comuns & $17(167)$ & $13(74)$ & 0,06 & $17(278)$ & $17(167)$ & 0,81 \\
\hline Hábito de fumar & $19(184)$ & $14(80)$ & 0,07 & $9(159)$ & $11(113)$ & 0,02 \\
\hline Consulta médica nos últimos 90 dias & $49(519)$ & $40(240)$ & 0,001 & $56(1.018)$ & $55(614)$ & 0,42 \\
\hline
\end{tabular}

PSF: Programa Saúde da Família; UBS: unidades básicas de saúde.

* Somente para os profissionais com especialização;

** Somente para quem referiu problemas de saúde.

(21\%) do que no Nordeste (19\%), no limiar da significância $(p=0,058)$. Em relação ao modelo de atenção, maiores prevalências foram registradas na amostra das UBS tradicionais, tanto do Sul (28\%) quanto no Nordeste $(23 \%)(\mathrm{p}<0,001)$.

Transtornos mentais comuns, aferidos pelo SRQ-20, estiveram presentes em $16 \%$ dos trabalhadores, sendo a prevalência semelhante entre os lotes e os modelos de atenção.
O hábito de fumar foi referido por $12 \%$ dos entrevistados, $17 \%$ no Sul e $10 \%$ no Nordeste ( $\mathrm{p}<0,001$ ), onde foi mais freqüente entre os trabalhadores das UBS tradicionais do Nordeste $(11 \% ; \mathrm{p}=0,02)$.

Mais de metade da amostra (52\%) consultou nos noventa dias anteriores à entrevista, sendo esta proporção maior no Nordeste (56\%) do que no Sul $(45 \%)(\mathrm{p}<0,001)$. 


\section{Discussão}

O estudo contribuiu de forma importante para o conhecimento das condições de trabalho e saúde de profissionais da Atenção Básica à Saúde em nosso país, considerando sua abrangência, sua estratificação por modelo assistencial e por região geopolítica.

A amostra por categoria profissional foi confrontada com o registro dos trabalhadores lotados na UBS, obtido no instrumento de avaliação da estrutura dos serviços 16. A menor cobertura foi observada para médicos da Região Nordeste (73\%). Para os demais profissionais dessa região, a média foi de $90 \%$, e no Sul foi de $93 \%$. Além disso, em favor da representatividade da amostra, os resultados descritos para profissionais do PSF estão de acordo com o perfil nacional revelado em 200219.

Mesmo considerando que as atribuições e a carga horária impliquem diferenciais entre os modelos de atenção, no Sul a maioria dos trabalhadores é mais bem remunerada do que no Nordeste, o que está de acordo com o perfil nacional para o PSF 19.

Os trabalhadores da atenção básica de saúde são em sua maioria mulheres entre 31 e 45 anos de idade. Esta realidade apresenta nuances diferentes quando analisada a partir do modelo de atenção, sendo a proporção de mulheres e dos mais jovens ainda maior no PSF.

Na composição da força de trabalho na atenção básica, a grande diferença entre os modelos reside na inclusão do agente comunitário da saúde na equipe do PSF. No entanto, no Nordeste, o início e a grande expansão do Programa de Agentes Comunitários de Saúde (PACS) tornaram a presença deste trabalhador bastante freqüente também na atenção tradicional.

Menos da metade dos trabalhadores ingressou por concurso público e um terço ou mais possuía vínculo precário. Em recente avaliação de tendências em recursos humanos 20 , a Rede Observatório de Recursos Humanos em Saúde da Organização Pan-Americana da Saúde destaca o entendimento dos profissionais de que formas diretas de vinculação trabalhista devam ser priorizadas.

Os resultados revelam que o trabalho precário foi ampliado a partir da expansão do PSF, de forma mais acentuada no Nordeste, onde a estratégia foi adotada mais precocemente e de maneira mais intensa. Ainda assim, aproximadamente dois terços dos profissionais estão satisfeitos com o vínculo de trabalho.

Embora dois terços tenham esse trabalho como único emprego, pouco mais de um terço tinha especialização na área, situação já destacada por Gil ${ }^{21}$ para médicos e enfermeiros vinculados ao PSF. A formação de recursos humanos para a atenção básica tem sido objeto de preocupação, tanto na esfera da gestão ${ }^{22}$, quanto da produção científica que, reconhecendo a importância do mercado de trabalho em saúde, destaca a necessidade de qualificação dos profissionais e de sua adequação aos novos modelos assistenciais 23,24,25,26,27.

A supervisão semanal do trabalho foi a realidade para apenas um terço desses trabalhadores, com conteúdos focalizados principalmente na produtividade. Os achados em relação ao conteúdo das supervisões está de acordo com a situação já problematizada em alguns estudos 28,29 de que o foco da supervisão em atenção básica ainda são os procedimentos e não os cuidados de saúde.

A metade dos trabalhadores entrevistados considera o ambiente físico do trabalho inadequado e quase dois terços têm opinião semelhante em relação às tarefas que desempenham e às relações institucionais. As relações pessoais foram consideradas insatisfatórias para um terço da amostra no Sul, enquanto no Nordeste esta proporção atinge $90 \%$ da amostra. Tais níveis são preocupantes, pois o trabalho é uma das fontes de satisfação de diversas necessidades humanas, como "auto-realização, manutenção das relações interpessoais e sobrevivência” 30 (p. 39). Em Pelotas, em 2004, cerca de um terço dos trabalhadores da rede básica avaliou suas condições de trabalho como inadequadas ${ }^{31}$. No caso dos serviços de saúde esse grau de insatisfação com as relações interpessoais pode ser um fator de comprometimento da qualidade do cuidado, afetando negativamente seu impacto na saúde da população.

A insatisfação com sua saúde e a referência feita a problemas na saúde foi a realidade para mais de um terço da amostra de trabalhadores. Os problemas cardio-circulatórios, osteomusculares e do tecido conjuntivo, bem como as doenças do aparelho digestivo e transtornos mentais comuns, aferidos pelo SRQ-20, foram os mais prevalentes nessa amostra de trabalhadores.

Apesar da possibilidade desses resultados sobre a situação de saúde terem sido subestimados pelo "efeito do trabalhador sadio" 32 , estão de acordo com estudos que têm verificado o impacto do trabalho na saúde 33 . Em relação aos transtornos mentais comuns, a prevalência encontrada de $16 \%$ foi inferior à observada em outros estudos com profissionais e estudantes da área da saúde 34,35,36,37, em estudos de base populacional e em outras categorias profissionais $38,39,40$.

As principais diferenças entre os modelos de atenção dizem respeito à constituição das equi- 
pes, sendo o PSF caracterizado por mais agentes comunitários de saúde, mais mulheres, profissionais mais jovens, menos ingresso por concurso, mais trabalhadores com um único emprego, maior precarização nos vínculos trabalhistas, menor satisfação com este vínculo, menor tempo de trabalho, maior carga horária, maior especialização na área e melhor remuneração.

Também deve-se destacar o aparente conflito entre o pior desempenho do PSF em algumas variáveis, dadas as peculiaridades do processo de trabalho sob a ótica da saúde da família, em que o trabalho em equipe demanda maior articulação entre seus membros, com mais supervisão e maiores níveis de cobrança.
Em relação às condições de saúde, diferenças importantes foram observadas na auto-avaliação da sua saúde e consultas médicas nos últimos três meses, ambos com piores desempenhos para os trabalhadores do PSF.

De modo geral, pode-se concluir que a realidade dos trabalhadores da atenção básica, desta amostra, independente do modelo de atenção ou da região, sugere uma grande lacuna a ser preenchida rumo ao alcance do que é preconizado, sendo ainda menos adequado na Região Nordeste e no modelo do PSF. Essa constatação é mais preocupante se for considerado que o PSF vem se afirmando como modelo substitutivo.

\section{Resumo}

Com o objetivo de descrever o perfil das equipes de saúde da atenção básica em 41 municípios com mais de 100 mil habitantes, um total de 4.749 trabalhadores de saúde de dois estados do Sul (1.730) e cinco do Nordeste (3.019) do Brasil foram incluídos a partir de amostra das unidades básicas de saúde tradicionais e do Programa Saúde da Família (PSF). Após consentimento, os trabalhadores responderam a um instrumento auto-aplicado com informações demográficas, sobre o trabalho e a sua situação de saúde. As principais diferenças entre os modelos de atenção foram na constituição das equipes, com o PSF apresentando mais agentes comunitários de saúde, mais mulheres, trabalhadores mais jovens, menor ingresso por concurso, mais traba- lhadores com um único emprego, maior precarização nos vínculos trabalhistas, menor satisfação com este vínculo, menor antiguidade no trabalho, maior carga horária, maior especialização na área e melhor remuneração. Pior avaliação de sua saúde e maior proporção de consultas médicas foram igualmente registradas para o PSF. São necessários esforços no âmbito da gestão para apoiar esses trabalhadores, que são a base do sistema de saúde e, protagonistas do seu desenvolvimento e da consolidação da atenção básica.

Atenção Básica à Saúde; Saúde da Família; Saúde do Trabalhador; Condições de Trabalho 


\section{Colaboradores}

L. A. Facchini, R. X. Piccini, E. Tomasi, E. Thumé, D. S. Silveira e V. A. Teixeira participaram da concepção, análise de dados, interpretação e redação final do artigo. F. V. Siqueira, M. A. Rodrigues eV. V. Paniz participaram da análise e interpretação dos dados, na revisão crítica e na redação final do manuscrito.

\section{Agradecimentos}

Os autores agradecem aos gestores, coordenadores de Atenção Básica à Saúde e do Programa Saúde da Família, representantes do controle social, trabalhadores das unidades básicas da saúde e à população entrevistada o apoio prestado à realização do estudo. Agradecem ainda ao empenho dos auxiliares de pesquisa e àqueles que de alguma maneira contribuíram para as diferentes etapas do trabalho. O presente artigo integra o Componente 3 do Projeto de Expansão e Consolidação do Saúde da Família, financiado pelo Ministério da Saúde e Banco Mundial.

\section{Referências}

1. Fassa AG, Facchini LA, Dall'Agnol MM. Trabalho e morbidade comum em indústria de celulose e papel: um perfil segundo setor. Cad Saúde Pública 1996; 12:297-307.

2. Faria NM, Facchini LA, Fassa AG, Tomasi E. Processo de trabalho rural e saúde na serra gaúcha: um estudo descritivo. Cad Saúde Pública 2000; 16:115-28.

3. Palácios M, Duarte F, Câmara VM. Trabalho e sofrimento psíquico de caixas de agências bancárias na Cidade do Rio de Janeiro. Cad Saúde Pública 2002; 18:843-51.

4. Pires D. Reestruturação produtiva e conseqüências para o trabalho em saúde. Rev Bras Enferm 2000; 53:251-63.

5. Peduzzi M, Palma JJL. A equipe de saúde. In: Schraiber LB, Nemes MIB, Mendes-Gonçalves $\mathrm{RB}$, organizadores. Saúde do adulto, programas e ações na unidade básica. São Paulo: Editora Hucitec; 1996. p. 234-50.

6. Metzner RJ, Fischer FM. Fadiga e capacidade para o trabalho em turnos fixos de doze horas. Rev Saúde Pública 2001; 35:548-53.
7. Molina-Siguero A, García-Pérez MA, AlonsoGonzález M, Carmeno CP. Prevalencia de desgaste profesional y psicomorbilidad en médicos de atención primaria de un área sanitaria de Madrid. Aten Primaria 2003; 31:564-71.

8. Prietto DAB. Avaliação de um programa de capacitação em saúde mental para médicos dos serviços básicos de saúde [Tese de Doutorado]. São Paulo: Escola Paulista de Medicina, Universidade Federal de São Paulo; 2004.

9. Hidalgo CG, Cabezas AM, Collados M. Clima organizacional en un centro de salud de atención primaria: evaluación e intervención para mejorar la satisfacción laboral del equipo de salud. Cuad Med Soc 1997; 38:3-12.

10. Pedrosa JIS, Teles JBM. Consenso e diferenças em equipes do Programa Saúde da Família. Rev Saúde Pública 2001; 35:303-11.

11. Organização Pan-Americana da Saúde/Ministério da Saúde. Chamado à ação de Toronto: 2006-2015. Rumo a uma década de recursos humanos em saúde nas Américas. Brasília: Ministério da Saúde; 2006. (Série D. Reuniões e Conferências). 
12. Ministério da Saúde. PROESF: Projeto de Expansão e Consolidação do Saúde da Família. Brasília: Ministério da Saúde; 2003.

13. Lemeshow S, Hosmer D. Adequacy of sample size in health studies. Chichester: John Wiley; 1990.

14. Levy P, Lemeshow S. Sampling for health professionals. Belmont: Lifetime Learning Publications; 1980.

15. Lwanga S, Lemeshow S. Sample size determination in health studies: a practical manual. Geneva: World Health Organization; 1991.

16. Facchini LA, Piccini RX, Tomasi E, Thumé E, Silveira DS. Relatório final do projeto de monitoramento e avaliação do Programa de Expansão e Consolidação da Saúde da Família (PROESF). Pelotas: Universidade Federal de Pelotas; 2006.

17. Figueroa NL, Schufer M, Muinos R, Marro C, Coria EA. Um instrumento para a avaliação de estressores psicossociais no contexto de emprego. Psicol Reflex Crit 2001; 14:653-9.

18. Mari JJ, Iacoponi E, Williams P, Simões O, Silva JBT. Distúrbios psiquiátricos e assistência primária à saúde no Brasil. Rev Saúde Pública 1987; 21:501-7.

19. Departamento de Atenção Básica, Secretaria de Atenção à Saúde, Ministério da Saúde. Avaliação normativa do Programa Saúde da Família no Brasil: monitoramento da implantação e funcionamento das equipes de saúde da família: 2001-2002. Brasília: Ministério da Saúde; 2004.

20. Nogueira RP, coordenador. Avaliação de tendências e prioridades sobre recursos humanos em saúde. Brasília: Organização Pan-Americana da Saúde; 2002. (Rede Observatório de Recursos Humanos em Saúde).

21. Gil CRR. Formação de recursos humanos em saúde da família: paradoxos e perspectivas. Cad Saúde Pública 2005; 21:490-8.

22. Ministério da Saúde. Princípios e diretrizes para a NOB/RH - SUS. Brasília: Conselho Nacional de Saúde, Ministério da Saúde; 2002.

23. Campos FE, Aguiar RAT. Atenção básica e reforma curricular. In: Negri B, Faria R, Viana ALD, organizadores. Recursos humanos em saúde: política, desenvolvimento e mercado de trabalho. Campinas: Editora da Unicamp; 2002. p. 91-9.

24. Bevilacqua RG, Sampaio SAP. As especializações: histórico e projeções. In: Negri B, Faria R, Viana ALD, organizadores. Recursos humanos em saúde: política, desenvolvimento e mercado de trabalho. Campinas: Editora da Unicamp; 2002. p. 33-90.

25. Cordeiro H. Os desafios do ensino das profissões de saúde frente às mudanças do modelo assistencial: contribuições para além dos Pólos de Capacitação. Ensaio: Avaliação de Políticas Públicas na Educação 2002; 10:43-54.

26. Pierantoni CR. Recursos humanos e gerência no SUS. In: Negri B, Faria R, Viana ALD, organizadores. Recursos humanos em saúde: política, desenvolvimento e mercado de trabalho. Campinas: Editora da Unicamp; 2002. p. 221-56.
27. Machado MH. Mercado de trabalho em saúde. In: Falcão A, Santos Neto PM, Costa PS, Belisário AS, organizadores. Observatório de recursos humanos em saúde no Brasil: estudos e análises. Rio de Janeiro: Editora Fiocruz; 2003. p. 227-48.

28. Matumoto S, Fortuna CM, Mishima SM, Pereira MJB, Domingos NAM. Supervisão de equipes no Programa de Saúde da Família: reflexões acerca do desafio da produção de cuidados. Interface Comun Saúde Educ 2005; 9:9-24.

29. Merhy EE. Em busca do tempo perdido: a micropolítica do trabalho vivo em saúde. In: Merhy EE, Onocko R, organizadores. Agir em saúde: um desafio para o público. São Paulo: Editora Hucitec; 1997. p. 71-112.

30. Murta SG, Troccoli BT. Avaliação de intervenção em estresse ocupacional. Psicol Teor Pesqui 2004; 20:39-47.

31. Tomasi E, Sant'Anna GC, Oppelt AM, Petrini RM, Pereira IV, Sassi BT. Condições de trabalho e automedicação em profissionais da rede básica de saúde da zona urbana de Pelotas, RS. Rev Bras Epidemiol 2007; 10:66-74.

32. Pereira MG. Epidemiologia: teoria e prática. Rio de Janeiro: Editora Guanabara Koogan; 1995.

33. Pinheiro FA, Troccoli BT, Paz MGT. Preditores psicossociais de sintomas osteomusculares: a importância das relações de mediação e moderação. Psicol Reflex Crit 2006; 19:142-50.

34. Veggi AB, Lopes CS, Faerstein E, Sichieri R. Índice de massa corporal, percepção do peso corporal e transtornos mentais comuns entre funcionários de uma universidade no Rio de Janeiro. Rev Bras Psiquiatr 2004; 26:242-7.

35. Nascimento Sobrinho CL, Carvalho FM, Bonfim TAS, Cirino CAS, Ferreira IS. Condições de trabalho e saúde dos médicos em Salvador, Brasil. Rev Assoc Med Bras 2006; 52:97-102.

36. Araújo TM, Aquino E, Menezes G, Santos CO, Aguiar L. Aspectos psicossociais do trabalho e distúrbios psíquicos entre trabalhadoras de enfermagem. Rev Saúde Pública 2003; 37:424-33.

37. Facundes VLD, Ludermir AB. Transtornos mentais comuns em estudantes da área de saúde. Rev Bras Psiquiatr 2005; 27:194-200.

38. Maragno L, Goldbaum M, Gianini RJ, Novaes HMD, César CLG. Prevalência de transtornos mentais comuns em populações atendidas pelo Programa Saúde da Família (QUALIS) no Município de São Paulo, Brasil. Cad Saúde Pública 2006; 22:1639-48.

39. Costa JSD, Menezes AMB, Olinto MTA, Gigante DP, Macedo S, Britto MAP, et al. Prevalência de distúrbios psiquiátricos menores na Cidade de Pelotas, RS. Rev Bras Epidemiol 2002; 5:164-73.

40. Ludermir AB, Melo Filho DA. Condições de vida e estrutura ocupacionalassociada a transtornosmentais comuns. Rev Saúde Pública 2002; 36:213-21.

Recebido em 22/Fev/2007

Aprovado em 09/Mai/2007 